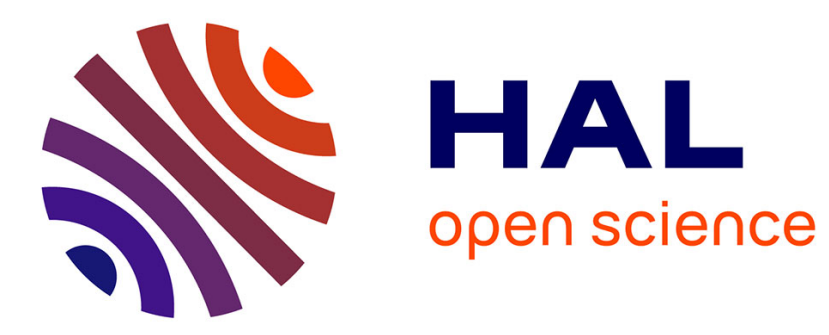

\title{
Central catadioptric image processing with geodesic metric
}

\author{
Cédric Demonceaux, Pascal Vasseur, Yohan Fougerolle
}

\section{To cite this version:}

Cédric Demonceaux, Pascal Vasseur, Yohan Fougerolle. Central catadioptric image processing with geodesic metric. Image and Vision Computing, 2011, 29 (12), pp.840-849. 10.1016/j.imavis.2011.09.007 . hal-00647150

\section{HAL Id: hal-00647150 \\ https://hal.science/hal-00647150}

Submitted on 30 Apr 2018

HAL is a multi-disciplinary open access archive for the deposit and dissemination of scientific research documents, whether they are published or not. The documents may come from teaching and research institutions in France or abroad, or from public or private research centers.
L'archive ouverte pluridisciplinaire HAL, est destinée au dépôt et à la diffusion de documents scientifiques de niveau recherche, publiés ou non, émanant des établissements d'enseignement et de recherche français ou étrangers, des laboratoires publics ou privés. 


\title{
Central Catadioptric Image Processing with Geodesic
}

\section{Metric}

\author{
Cédric Demonceaux ${ }^{1 *}$ Pascal Vasseur $^{2}$ Yohan Fougerolle $^{1}$ \\ ${ }^{1}$ LE2I-UMR 5158, University of Burgundy, 12 rue de la fonderie, 71200 Le Creusot, \\ France. \\ ${ }^{2}$ LITIS, Université de Rouen, 76800 Saint Etienne du Rouvray
}

\begin{abstract}
Because of the distortions produced by the insertion of a mirror, catadioptric images can not be processed similarly to classical perspective images. Now, although the equivalence between such images and spherical images is well known, the use of spherical harmonic analysis often leads to image processing methods which are more difficult to implement. In this paper, we propose to define catadioptric image processing from the geodesic metric on the unitary sphere. We show that this definition allows to adapt very simply classical image processing methods. We focus more particularly on image gradient estimation, interest point detection, and matching. More generally, the proposed approach extends traditional image processing techniques based on Euclidean metric to central catadioptric images. We show in this paper the efficiency of the approach through different experimental results and quantitative evaluations.
\end{abstract}

Key words:

Catadioptric image, image processing, spherical image

* Corresponding author. Tel : +33-385-731-128.

Email addresses: cedric.demonceaux@u-bourgogne.fr (Cédric 


\section{Introduction}

More and more applications benefit from the use of large or panoramic field of view cameras [1]. Indeed, a $360^{\circ}$ view of the camera environment is a very rich source of informations in order to perform tasks such as obstacle avoidance, occupancy map estimation, or beacon tracking even for six degrees of freedom systems... Many different solutions have been proposed in order to obtain panoramic images such as rotating camera, camera network, or the association of a mirror and a classical camera (fig 1). The latter option is often chosen because it provides an omnidirectional image in a single shot and can then be used in dynamic environment. Consequently, this kind of sensor is now ubiquitously used in mobile robotic for tasks such as navigation, surveillance, visual simultaneous localization and mapping (SLAM) [2],[3]. However, because of the distortions observed in such catadioptric images (fig 1), traditional image processing techniques are no longer appropriate and require to be adapted to the new sensor geometry. As shown in figure (fig 2), the influence of a pixel on its neighbours strongly depends on its position in the image. Thus, while the distance between $P 1$ and $P 2$ and between $P 3$ and $P 4$ is equal, it appears that pixel $P 2$ does not have the same impact on $P 1$ as $P 4$ on $P 3$ because of the distortions induced by the mirror. In this way, classical operators can not be directly applied to this type of images.

In order to take into account the distortions implied by the sensor during the omnidirectional image processing, the intrinsic parameters have to be considered and used for defining a new representation space. In [4], Bogdanova et al. propose to

$\left.\overline{\text { Demonceaux }}^{1}\right)$, Pascal.Vasseur@univ-rouen. fr (Pascal Vasseur $\left.{ }^{2}\right)$, yohan.fougerolledu-bourgogne.fr(Yohan Fougerolle $\left.{ }^{1}\right)$. 


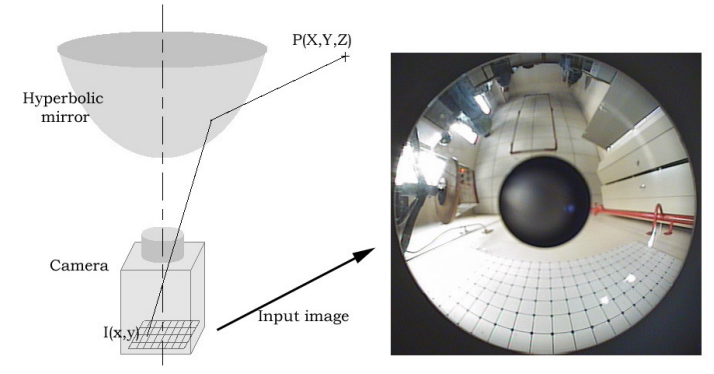

Fig. 1. Catadioptric camera and catadioptric image

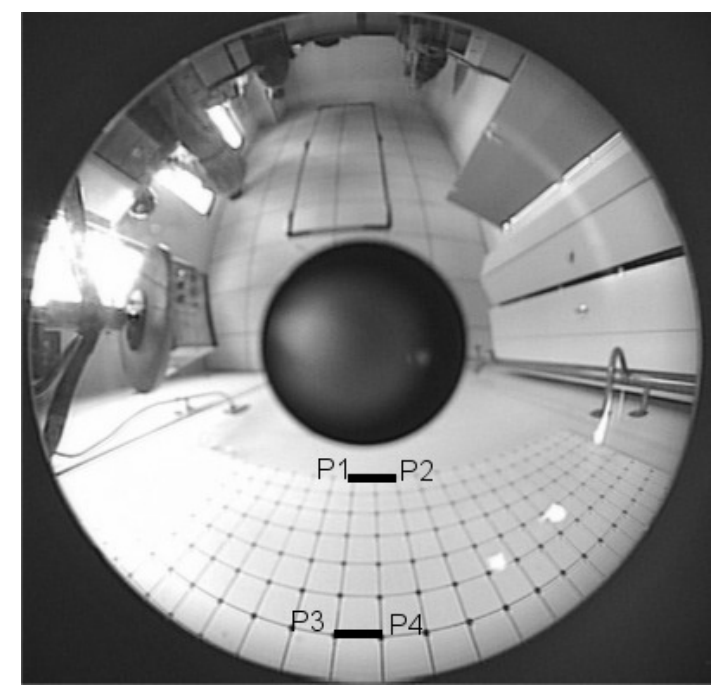

Fig. 2. $d(P 1, P 2)=d(P 3, P 4)$ when $P 1$ does not have physically the same influence on $P 2$ than $P 3$ on $P 4$.

perform the image processing directly in the image space by taking into account the deformation due to the mirror thanks to the Jacobian induced by the mirror geometry. While this approach corrects the image distortions during the gradient estimation, it can not be extended to image filtering. Indeed, in this case, the metric change equations allow to consider the new geometry of the space and consequently to obtain the associated differential operator formula by the Jacobian. However, this space change can not be applied to filtering operators which are defined by a convolution product. The unitary sphere represents an other space which is particularly useful for omnidirectional image processing. As demonstrated in [5] and [6], there exists an equivalence between the unitary sphere and single view point (SVP), also 
called central images and even some fisheye images [7]. However, this equivalence is related to the knowledge of the intrinsic parameters of the sensor (fig 3) and can be decomposed into a double projection as follows: 3D point $P_{w}$ is projected on the sphere surface according to the sphere center in $P_{s}$ which is then projected on the image plane into $P_{i}$ from the projection center $O_{p}$ located between the center and the north pole of the sphere. The position of $O_{p}$ depends on the type of sensor.

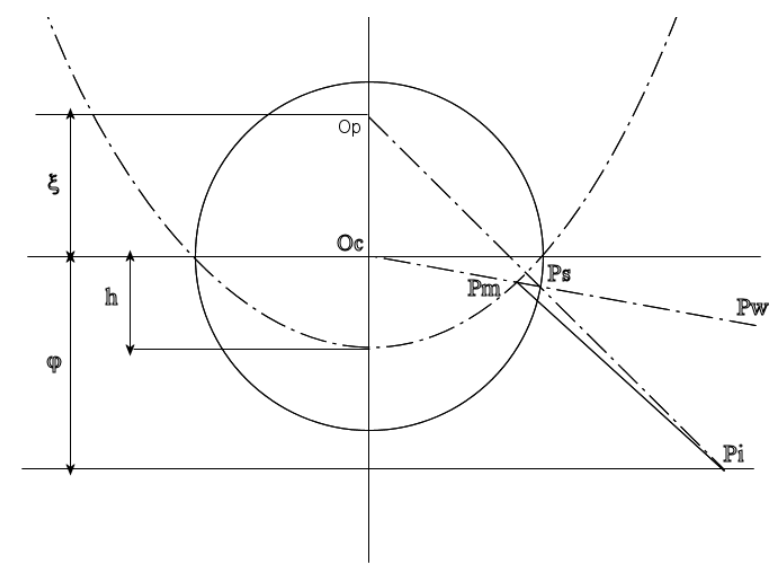

Fig. 3. Equivalence between the catadioptric projection and the two-step mapping via the sphere.

This spherical space, which appears to be more natural for central images than Euclidean space, has been considered either for the processing directly, as detailed in the next section or as an intermediate space for a new representation. For example, in [8], the authors proposed to project the central image onto a virtual cylinder in order to avoid the spherical geometry constrains. By considering that this cylinder is locally planar, they apply directly conventional filtering operators. Nevertheless, even if this representation can be considered as a valid approximation, the geometry on the cylinder is not appropriate to spherical images. Indeed, this cylindrical modeling defines operators that are anisotropic on the spherical image and then leads to different operations according to the pixel position in the catadioptric image. In [9], Demonceaux and Vasseur developed a neighborhood based on the spherical 
dependency of each image pixel. They proposed to use this approach in order to adapt Markovian methods to omnidirectional images. This representation shows a significant improvement of the Markovian methods but can not be generalized to image filtering since the number of neighbors of one pixel depends on its position in the image. The approach proposed in this paper is based on a direct processing on the sphere. We propose in the next section a presentation of the existing related works in order to bring out the differences and the contributions of our approach.

\section{Spherical Image Processing: an overview}

In order to process central omnidirectional images, some authors ([10], [11], [12], [4]) propose to consider them as spherical images and develop specifically dedicated tools for such images. They used the spherical harmonic analysis in order to define the convolution and the Fourier analysis. We develop such different methods to provide clear comparison with our approach. Precisely, we describe the different steps required to evaluate the spherical gradient and to filter such image.

\subsection{Spherical Gradient}

The unit sphere $\mathcal{S}^{2}$ can be parameterized by spherical coordinates:

$$
\forall x \in \mathcal{S}^{2}, x=(\cos (\phi) \sin (\theta), \sin (\phi) \sin (\theta), \cos (\theta))
$$

where $\phi \in[0,2 \pi[, \theta \in[0, \pi]$.

With this parameterization, the spherical gradient is defined by:

$$
\nabla_{\mathcal{S}^{2}} I(\theta, \phi)=\frac{\partial I}{\partial \theta} e_{\theta}(\theta, \phi)+\frac{1}{\sin (\theta)} \frac{\partial I}{\partial \phi} e_{\phi}(\theta, \phi) .
$$


Thus, if we adopt a regular sampling of the sphere based on $\theta_{i}$ and $\phi_{j}$, the spherical gradient can be computed thanks to finite differences [11] as:

$$
\begin{aligned}
\left\|\nabla_{\mathcal{S}^{2}} I\left(\theta_{j}, \phi_{k}\right)\right\|^{2}= & \left|I\left(\theta_{j+1}, \phi_{k}\right)-I\left(\theta_{j}, \phi_{k}\right)\right|^{2} \\
& +\frac{1}{\sin ^{2}\left(\theta_{j}\right)}\left|I\left(\theta_{j}, \phi_{k+1}\right)-I\left(\theta_{j}, \phi_{k}\right)\right|^{2} .
\end{aligned}
$$

We can note that the formulation of the spherical gradient depends of the latitude $\theta$ because the distance between the points $\left(\theta_{j}, \phi_{k}\right)$ is not regular (fig. 4). This problem more clearly appears when we want to compute a discrete Laplace operator:

$$
\triangle_{S^{2}}=\frac{1}{\sin \theta} \frac{\partial}{\partial \theta}\left(\sin \theta \frac{\partial}{\partial \theta}\right)+\frac{1}{\sin ^{2} \theta} \frac{\partial^{2}}{\partial^{2} \phi} \text {. }
$$

In order to avoid the computation of differential operators on the sphere, Bogdanova et al. [4] proposed to consider the equivalent projection between the catadioptric image plane and the spherical image proposed by [6]. For example, for a paracatadioptric image which is equivalent to a spherical image by stereographic projection, the metric induced on the sphere is equivalent to:

$$
d l_{S^{2}}=d \theta^{2}+\sin ^{2} \theta d \phi^{2}=\frac{4}{\left(1+x^{2}+y^{2}\right)^{2}}\left(d x^{2}+d y^{2}\right)
$$

Thus, the spherical gradient can be computed thanks to the classical Euclidean gradient by multiplying with a scaling factor:

$$
\nabla_{\mathcal{S}^{2}}=\frac{\left(1+x^{2}+y^{2}\right)^{2}}{4} \nabla_{\mathbb{R}^{2}}
$$

The main advantage of this formulation is that we can compute the image gradient directly in the planar image with cartesian coordinates while respecting the non planar geometry of the mirror. However, the equation (6) is valid only for a parabolic miror and the formulation is different if we consider an hyperbolic mirror. 


\subsection{Spherical Filtering and Harmonic Analysis}

On the sphere, the classical convolution used in Euclidean metric is not valid. Driscoll and Healy [13] have developed new tools based on spherical harmonics. Let us note $<., .>$ the inner product on the sphere:

$$
f, g \in L^{2}\left(\mathcal{S}^{2}\right), \quad<f, g>=\int_{0}^{\pi} \int_{0}^{2 \pi} f(\theta, \phi) \overline{g(\theta, \phi)} \sin \theta d \theta d \phi
$$

The spherical harmonics $Y_{l}^{m}(\theta, \phi)$, defined by Legendre polynomials, form an orthonormal basis of $L^{2}\left(\mathcal{S}^{2}\right)$, and a function $f$ can be decomposed by (see [13]):

$$
f=\sum_{l \geqslant 0} \sum_{|m| \leqslant l} \hat{f}(l, m) Y_{l}^{m}(\theta, \phi) \quad, \text { where } \quad \hat{f}(l, m)=<f, Y_{l}^{m}>
$$

Driscoll and Healy define the spherical convolution as:

$$
f, g \in L^{2}\left(\mathcal{S}^{2}\right), \quad(f * g)(\eta)=\int_{S O(3)} f\left(R n_{0}\right) g\left(R^{-1} \eta\right) d R
$$

where $\eta \in \mathcal{S}^{2}, n_{0}=(0,0,1)$ is the North of the sphere. Similar definition can be seen in [14] and [15].

This convolution can be computed in Fourier domain thanks to the convolution theorem:

$$
f, g \in L^{2}\left(\mathcal{S}^{2}\right), \quad(\widehat{f * g})(l, m)=2 \pi \sqrt{\frac{4 \pi}{2 l+1}} \hat{f}(l, m) \hat{g}(l, 0) .
$$

With these notations, the spherical Gaussian function $G$ (which solves the spherical diffusion equation $\left.\triangle_{S^{2}} u=\partial_{t} u\right)$ is given by:

$$
\widehat{G(., t)}(l, m)= \begin{cases}\sqrt{\frac{2 l+1}{4 \pi}} e^{-l(l+1) t} & \text { if } \quad m=0 \\ 0 & \text { else }\end{cases}
$$




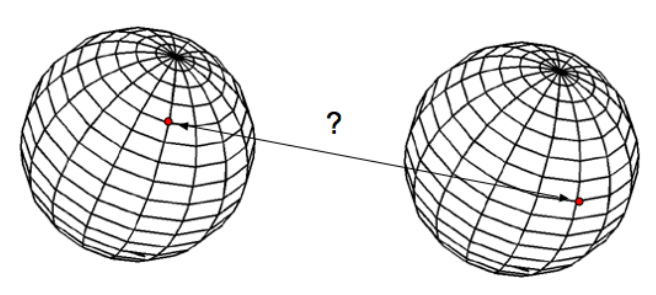

Fig. 4. Sampling of the sphere. This irregular sampling is unappropriate for the matching by Zero mean Normalized Cross-Correlation function (ZNCC).

So, we can smooth a spherical image using (11), (10), and (8). Nevertheless, we can note that the spherical convolution is not commutative $(f * g \neq g * f)$ and does not verify the derivative relation which is very useful for edge detection in perspective image $(f * g)^{\prime}=f^{\prime} * g=f * g^{\prime}[11]$. Thus, the image gradient can not be computed by using a low-pass filter as it is commonly done in perspective image processing.

In this paper, we propose a new definition of the neighborhood between pixels of a central catadioptric image. We show that this definition allows to use the same operators than in classical image processing techniques. In the next section, we examine the necessity of modifying the notion of neighborhood in catadioptric images and present the choice of the geodesic metric. The section 4 is dedicated to the definition of the new convolution product and its use in catadioptric image filtering. Different experimental results on edge detection and interest point detection will be presented and evaluated. Point matching methods derived from the new neighborhood definition will be proposed in Section 5 before a conclusion. 


\section{Metric and Geodesic Neighborhood}

\subsection{Introduction}

In perspective image processing, most methods are developed from Euclidean metric:

$$
\forall x, y \in \mathbb{R}^{2}, d_{\mathbb{R}^{2}}(x, y)=\|x-y\|_{l^{2}\left(\mathbb{R}^{2}\right)} .
$$

From this metric, a neighborhood can be derived to define pixels dependency between each other. Derivate computation, corner detection, or point matching by correlation can then be performed thank this neighborhood definition. Theoretically, the neighborhood expresses the mutual 3D point influence into the image plane. Practically, if we consider an orthographic camera, the Euclidean neighborhood exactly represents 3D points placed on a fronto-parallel plane (fig 5(a)). In the case of a perspective camera, this neighborhood is generally considered as a sufficiently good approximation whatever the 3D point configuration (fig 5(b)). Considering a central catadioptric image, the Euclidean neighborhood is no longer relevant (fig 5(c)). However, a regular neighborhood according to $\theta$ and $\phi$ angles (fig 5(d)) will translate exactly mutual 3D point influence if they are placed on a concentric sphere of the unitary sphere. This is the kind of neighborhood that we develop in the following. 


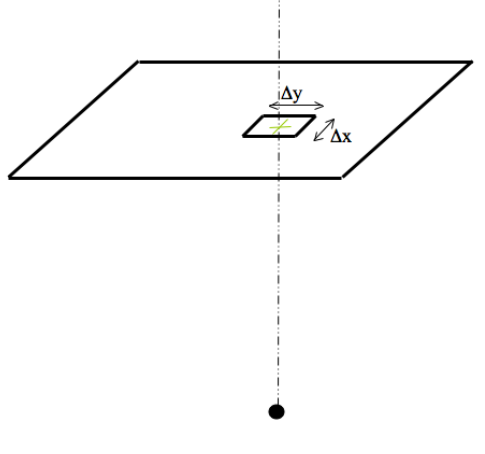

(a)

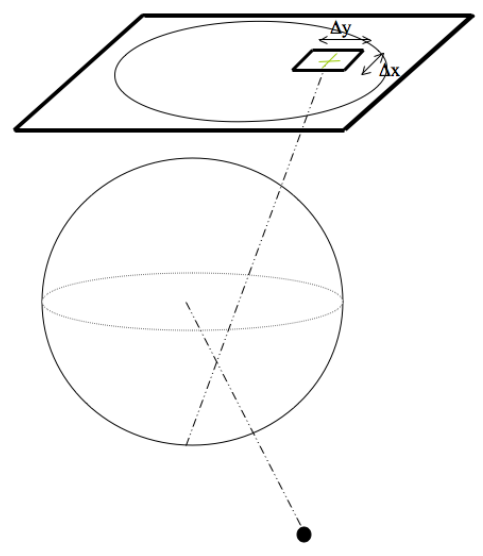

(c)

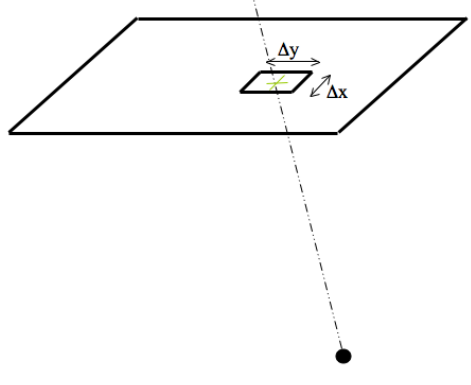

(b)

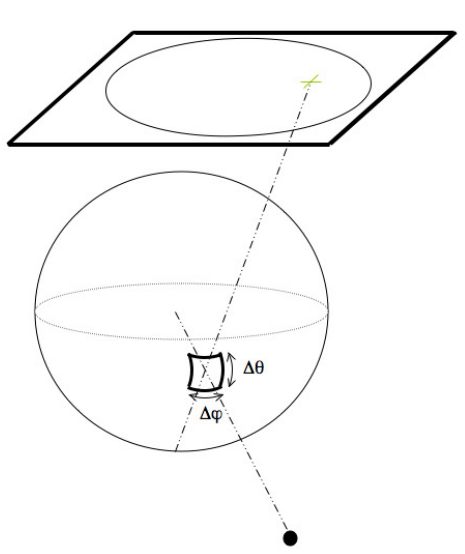

(d)

Fig. 5. Image formation and neighborhood dependency. (a) Orthographic image, Euclidean metric, (b) perspective image, Euclidean metric, (c) omnidirectional image, Euclidean metric, (d) omnidirectional image, geodesic metric.

\subsection{Neighborhood Definition}

Let note $\mathrm{P}$ the projection that transforms a central catadioptric image which belongs to $\mathbb{R}^{2}$ into his equivalent spherical image on $\mathcal{S}^{2}$ :

$$
\begin{aligned}
\mathrm{P}: & \mathbb{R}^{2} \rightarrow \quad \mathcal{S}^{2} \\
x & \mapsto x_{s}=(\theta, \phi)
\end{aligned}
$$




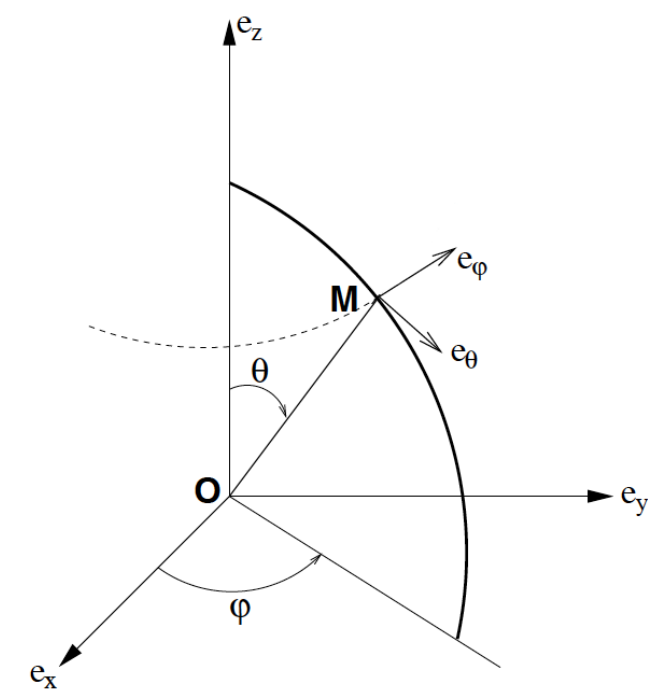

Fig. 6. Spherical coordinates and local basis.

In order to process spherical images, we propose to use the following geodesic distance:

$$
\begin{aligned}
& \forall x_{s}=(\theta, \phi), y_{s}=\left(\theta^{\prime}, \phi^{\prime}\right) \in \mathcal{S}^{2} \\
& d_{\mathcal{S}^{2}}(x, y)=\operatorname{arcos}\left[\left(\left[\begin{array}{c}
\cos (\phi) \sin (\theta) \\
\sin (\phi) \sin (\theta) \\
\cos (\theta)
\end{array}\right)^{T} \cdot\left(\begin{array}{c}
\cos \left(\phi^{\prime}\right) \sin \left(\theta^{\prime}\right) \\
\sin \left(\phi^{\prime}\right) \sin \left(\theta^{\prime}\right) \\
\cos \left(\theta^{\prime}\right)
\end{array}\right)\right] .\right.
\end{aligned}
$$

Let note $x_{s} \in \mathcal{S}^{2}$ the projection of an image pixel $x$ onto the unitary sphere $\left(x_{s}=\right.$ $\mathrm{P}(x)$ ). We then define the continuous neighborhood $\mathcal{V}_{r}(x)$ of pixel $x$ in the image as follows:

$$
\mathcal{V}_{r}(x)=\left\{y_{s} \in \mathcal{S}^{2}, d_{\mathcal{S}^{2}}\left(x_{s}, y_{s}\right) \leqslant r \mid \mathrm{P}(x)=x_{s}\right\}
$$

This neighborhood results from the intersection between the cone with apex $O$, direction $O x_{s}$, angle $r$, and the sphere $\mathcal{S}^{2}$. In order to use discrete convolution filters with this neighborhood, it is then necessary to introduce a discrete version of 
(14). In this way, we consider the tangent plane $\pi$ to $\mathcal{S}^{2}$ at $x_{s}$. This plane has the following equation $x_{s}+a \vec{e}_{\theta}+b \vec{e}_{\phi}, \quad(a, b) \in \mathbb{R}^{2}$, where $\left(\vec{e}_{\theta}, \vec{e}_{\phi}\right)$ are vectors of the basis at $x_{s}=(\theta, \phi)$ (fig 6) :

$$
\vec{e}_{\theta}=\frac{\partial O \vec{M}}{\partial \theta}=\left(\begin{array}{c}
\cos \phi \cos \theta \\
\sin \phi \cos \theta \\
-\sin \theta
\end{array}\right), \quad \vec{e}_{\phi}=\frac{1}{\sin \theta} \frac{\partial O \vec{M}}{\partial \phi}=\left(\begin{array}{c}
-\sin \phi \\
\cos \phi \\
0
\end{array}\right)
$$

Since we want a regular mesh with constant geodesic distance, we obtain $(2 N+1)^{2}$ points of $\pi$ as follows :

$$
x_{s}+\tan (n r) \vec{e}_{\theta}+\tan (p r) \vec{e}_{\phi}, \quad-N \leq n, p \leq N
$$

i.e. $(2 N+1)^{2}$ points of $\mathcal{S}^{2}$ as:

$$
\mathcal{V}_{r}^{N}(x)=\left\{x_{s}(n, p)=\frac{x_{s}+\tan (n r) \vec{e}_{\theta}+\tan (p r) \vec{e}_{\phi}}{\left\|x_{s}+\tan (n r) \vec{e}_{\theta}+\tan (p r) \vec{e}_{\phi}\right\|}, \quad-N \leq n, p \leq N, x_{s}=\mathrm{P}(x)\right\}
$$

In $\mathcal{V}_{r}^{N}(x)$, the points are all equidistant in term of geodesic metric:

$$
\forall y \in \mathcal{V}_{r}^{N}(x), \quad \min _{z \in \mathcal{V}_{r}^{N}(x) \backslash y} d_{\mathcal{S}^{2}}(y, z)=r
$$

Note that this geodesic neighborhood depends on $r$. This value corresponds to the distance between two points of the neighborhood. In order to compare our results with conventional methods, we will set $r$ such that the geodesic neighborhood is equivalent to the Euclidean neighborhood in the center of the catadioptric image where the distortions are negligible. 


\subsection{Application to Catadioptric Image Processing}

In order to demonstrate the validity of the approach, we propose to adapt some conventional image processing methods by using the geodesic metric. We show that this metric allows to apply the classical methods to catadioptric images very easily. However, it is worth noting that practically, this new neighborhood requires that the luminance of each point to be known. In order to avoid a computationally expensive interpolation method for each point $\left(x_{s}(n, p)\right)$, we propose to use a simple nearest neighbour approach. We show that our method significantly improves the results, even with such rough interpolation.

First, we focus on linear catadioptric image filtering by defining the convolution product from the geodesic metric. We propose to apply this filtering for derivates computation and Harris corner detection. Then, we will see that feature matching methods based on similarity can also be adapted to central catadioptric images.

\section{Linear Catadioptric Image Filtering}

Linear image filtering consists in applying a weighted mask in each image point through convolution product. According to the previously mentioned reasons, the conventional convolution product can not be applied to catadioptric images. Consequently, the first step consists in defining the convolution product based on geodesic metric in order to process every pixel of the catadioptric image. We show that this new definition implies that the conventional operators remain valid. 


\subsection{Convolution}

Let consider an omnidirectional image $I$ and let note $I_{\mathcal{V}_{r}^{N}(x)}=I\left(\mathrm{P}^{-1}\left(\mathcal{V}_{r}^{N}(x)\right)\right)$, where $I_{\mathcal{V}_{r}^{N}(x)}$ represents the $(2 N+1)^{2}$ grey level values of the regular grid centered at $x_{s}=\mathrm{P}(x)$ (where $\mathrm{P}$ is defined by (13)).

$$
I_{\mathcal{V}_{r}^{N}(x)}(n, p)=I\left(\mathrm{P}^{-1}\left(x_{s}(n, p)\right)\right) \quad-N \leq n, p \leq N .
$$

Let $H$ be a filter with size $(2 N+1) \times(2 N+1)$, we define the convolution product of image $I$ by filter $H$ on point $x \in \mathbb{R}^{2}$ as follows:

$$
\begin{aligned}
\forall x \in \mathbb{R}, I H(x) & =I_{\mathcal{V}_{r}^{N}(x)} * H(x) \\
& =\sum_{i=-N}^{N} \sum_{j=-N}^{N} I_{\mathcal{V}_{r}^{N}(x)}(i, j) H(i, j) .
\end{aligned}
$$

\subsection{Geodesic Neighborhood vs Euclidean Neighborhood on the Tangent Plane}

It is also possible to define a regular sampling at $x_{s}$ on the tangent plane $\pi$ by using the distance $d_{\mathbb{R}^{2}}(x, y)$. This neighborhood is given by:

$$
\mathcal{V}_{r, \mathbb{R}^{2}}^{N}(x)=\left\{x_{s}+\tan (r)\left(n \vec{e}_{\theta}+p \vec{e}_{\phi}\right), \quad-N \leq n, p \leq N\right\}
$$

In $\mathcal{V}_{r, \mathbb{R}^{2}}^{N}(x)$, points are equidistant in term of Euclidean distance:

$$
\forall y_{\pi} \in \mathcal{V}_{r, \mathbb{R}^{2}}^{N}(x), \quad \min _{z_{\pi} \in \mathcal{V}_{r, \mathbb{R}^{2}}^{N}(x) \backslash y_{\pi}} d_{\mathbb{R}^{2}}\left(y_{\pi}, z_{\pi}\right)=\tan r
$$

$\mathcal{V}_{r, \mathbb{R}^{2}}^{N}(x)$ and $\mathcal{V}_{r}^{N}(x)$ are obviously different because $\tan n r \neq n \tan r$. Nevertheless, if we consider a viewing angle equal to $r=5^{\circ}$, which corresponds to a sampling of the sphere in $180 / 5 \times 360 / 5=36 \times 72$ pixels and a neighborhood of 


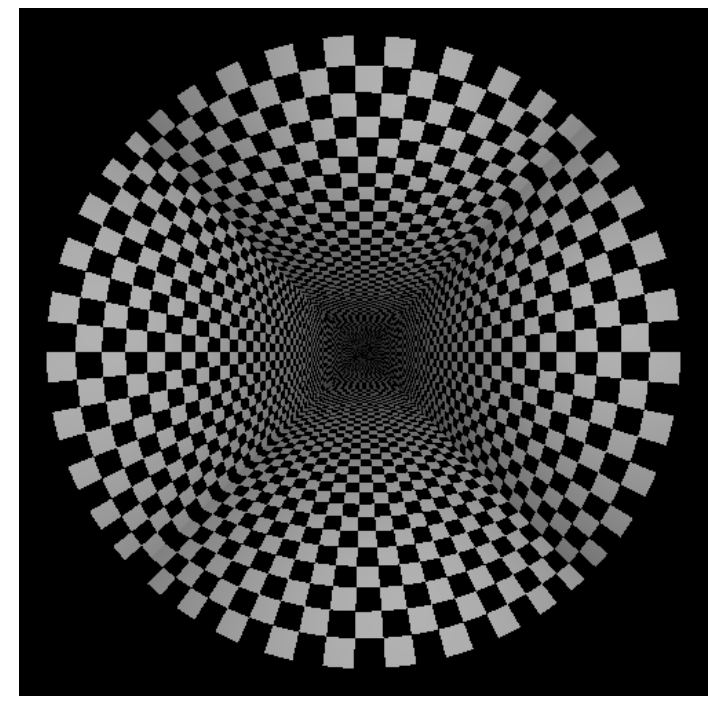

Fig. 7. Synthetic catadioptric test image.

size $9 \times 9$, the both extreme points of $\pi$ according to the neighorhoods $\mathcal{V}_{r, \mathbb{R}^{2}}^{N}(x)$ and $\mathcal{V}_{r}^{N}(x)$ are less than 0.02 pixel distant. This is why the geodesic neighborhood can be considered as a valid approximation of the spherical projection of the tangent plane Euclidean neighborhood. Consequently, the classical filters used on the tangent plane can be applied directly on the geodesic neighborhood.

\subsection{Derivative Filter}

According to the previous considerations, the derivative in $x_{s}$ can be computed by a classical Sobel filter such as:

$$
\|\left.\nabla I\left(x_{s}\right)\right|^{2} \simeq\left|I_{\mathcal{V}_{r}^{1}(x)} * S\right|^{2}+\left|I_{\mathcal{V}_{r}^{1}(x)} * S^{T}\right|^{2}
$$


where

$$
S=\left(\begin{array}{ccc}
-1 & -2 & -1 \\
0 & 0 & 0 \\
1 & 2 & 1
\end{array}\right)
$$

In order to verify the behaviour of this geodesic filtering, we applied it to a synthetic image (fig 7) and compared the results with the classical Sobel filter (fig 8) and with the Bogdanova's method (6). This synthetic image simulates a catadioptric camera placed inside a rectangular parallelepiped. This configuration is not optimal for the geodesic neighborhood. Indeed, as mentioned before, the geodesic neighborhood is optimal for the case of a spherical scene captured from its center by a catadioptric camera. However, we can note a slight qualitative improvement of the gradient computation. We more clearly distinguish the edges in the center of the image and their thickness is more regular along the radial lines in the geodesic approach (fig 8(f)). Note that Bogdanova's approach globally improves the detection but reduces the detection of lines near the image center Fig. 8(c)). This phenomenon is more clearly visible in the real case image in (Fig. 9(a)). Figure 9 presents the gradient computation in the case of a real image (fig 9(a)). In these results, we consider only pixels with a gradient norm greater than half times the maximum gradient value. It is important to note that the adapted gradient provides better results than the classical method essentially on the periphery of the image where the edges are qualitatively more precisely detected.

We have illustrated that the geodesic distance improves gradient computation for catadioptric images and that the obtained results are comparable to Bogdanova's method which can not be directly adapted to other filtering techniques, whereas our 


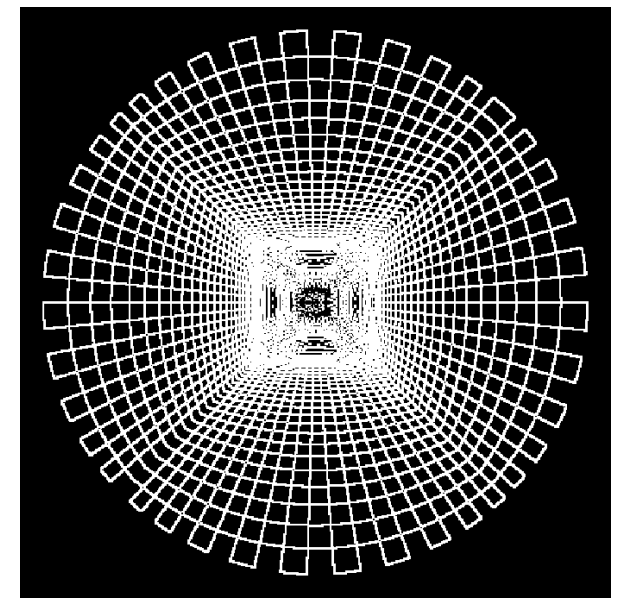

(a)

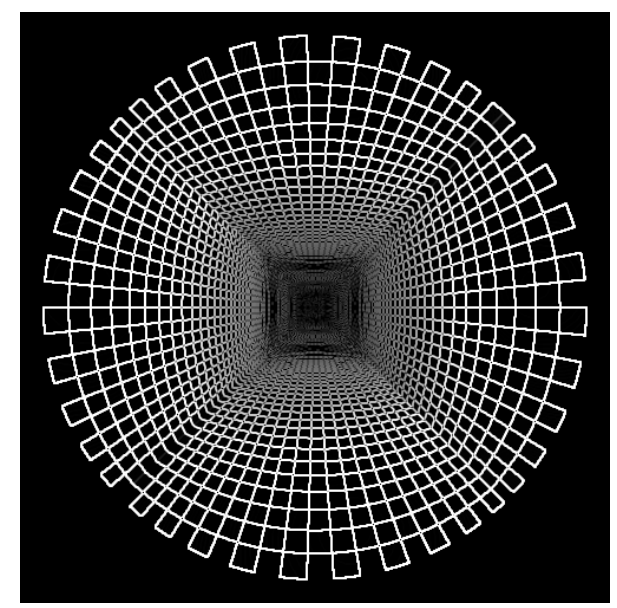

(c)

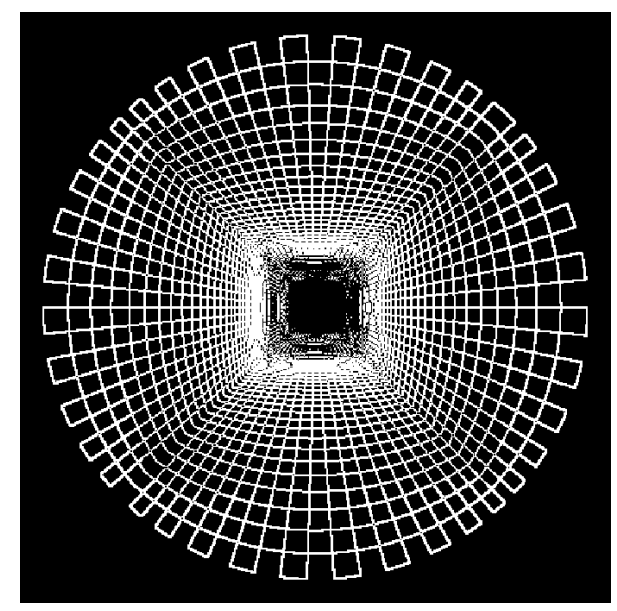

(e)

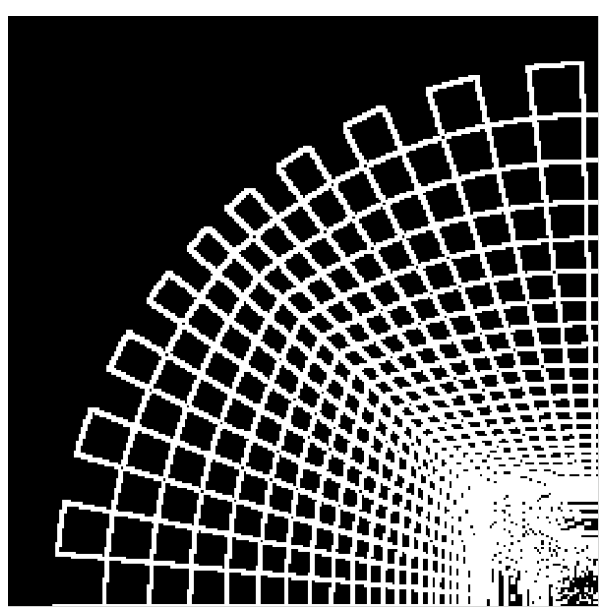

(b)

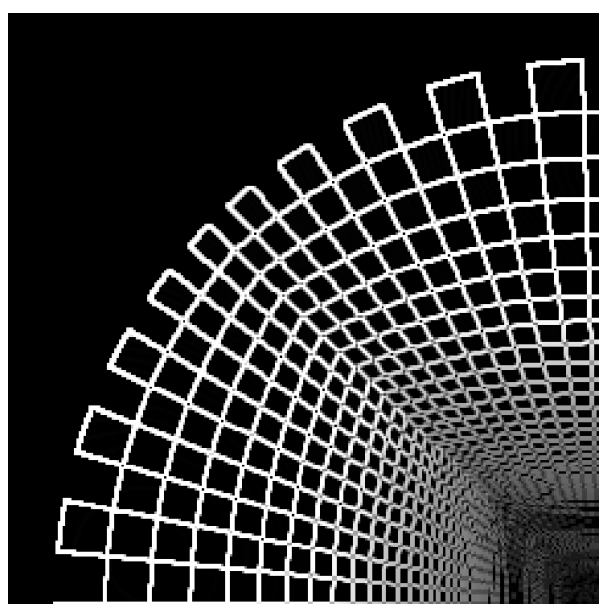

(d)

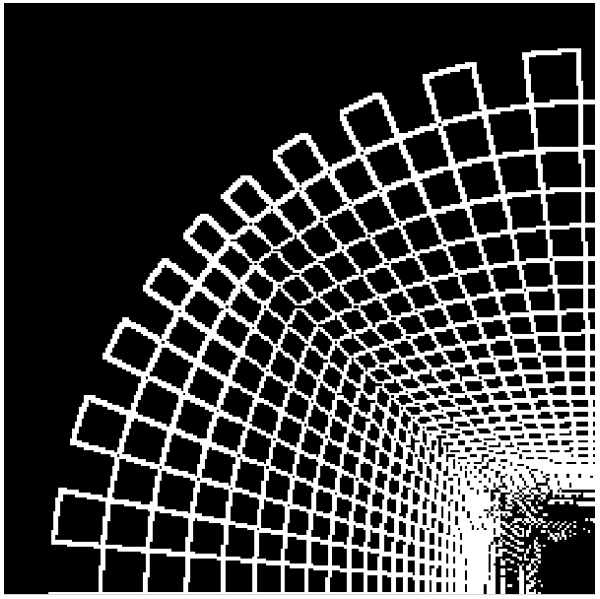

(f)

Fig. 8. Gradient norm results on synthetic image. (a),(b) Classical approach, (c) (d) Bogdanova's method [4], (e)(f) Geodesic approach 


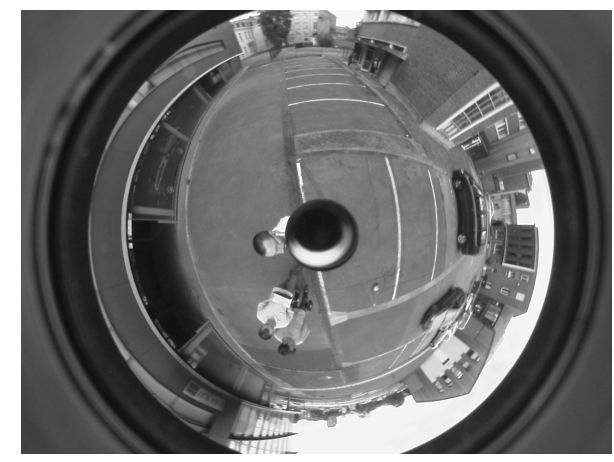

(a)

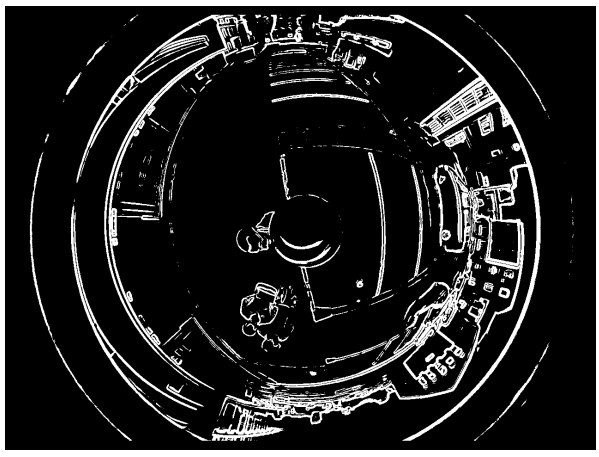

(c)

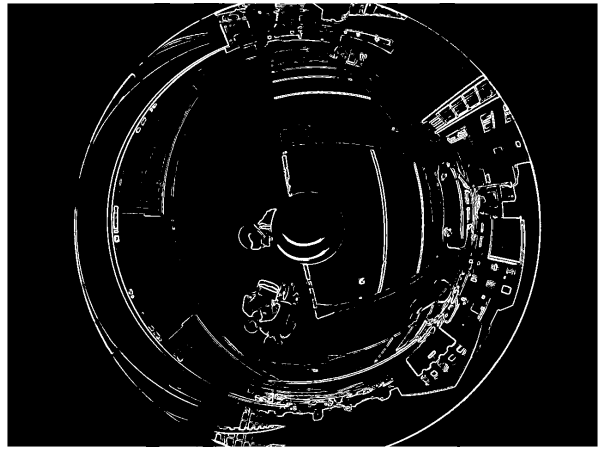

(b)

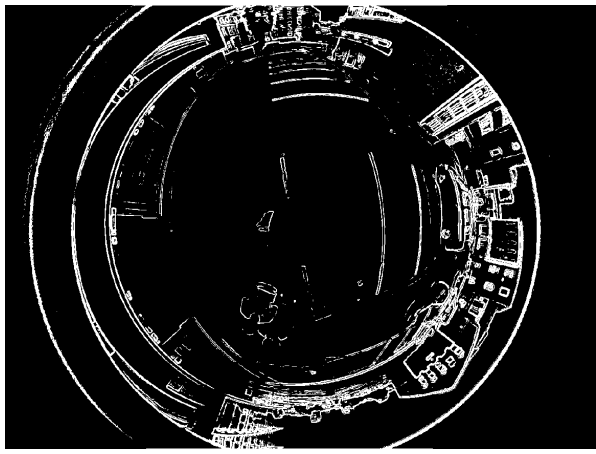

(d)

Fig. 9. Gradient norm results on real image. (a) Initial image, (b) Classical approach, (c) Geodesic approach, (d) Bogdanova's method [4]

approach remains valid in a more general framework.

\subsection{Gaussian and Laplacian Filters}

Similarly to the previous case, Gaussian and Laplacian filters remain valid with the geodesic neighborhood. Indeed, we can consider the Gaussian filtering as a weighting of the points according to their distance with the central point. This leads to substitute the Gaussian:

$$
G_{x}(y, \sigma)=\frac{1}{2 \pi \sigma^{2}} e^{-\frac{\|x-y\|^{2}}{2 \sigma^{2}}} \quad(x, y) \in \mathbb{R}^{2}
$$




$$
G_{x}(y, \sigma)=\frac{1}{2 \pi \sigma^{2}} e^{-\frac{d_{\mathcal{S}} 2(x, y)}{2 \sigma^{2}}} \quad(x, y) \in \mathcal{S}^{2} .
$$

The Laplacian of Gaussian can also be defined as:

$$
L o G_{x}(y)=-\frac{1}{\pi \sigma^{4}}\left[1-\frac{d_{\mathcal{S}^{2}}(x, y)}{2 \sigma^{2}}\right] \exp -\frac{d_{\mathcal{S}^{2}}(x, y)}{2 \sigma^{2}}
$$

Thus, while the Gaussian or Laplacian of Gaussian definitions using spherical harmonic analysis for spherical image processing needs their own rewording (11) [14][15], our approach provides a straightforward adaptation to such conventional filters. Indeed, thanks to $(19,20)$ and the convolution product defined in $(17)$, the masks used in perspective image processing remain valid.

In the following, we propose to verify the behaviour of these filters by the application of the Harris corner detector [16]. Harris corner detector has been massively used in computer vision applications. In perspective image processing, the corner detection by Harris method consists in studying the eigenvalues of matrix $M$ in each point $X$ :

$$
M(X)=\left[\begin{array}{cc}
L_{x}^{2}(X, \sigma) & L_{x} L_{y}(X, \sigma) \\
L_{x} L_{y}(X, \sigma) & L_{y}^{2}(X, \sigma)
\end{array}\right],
$$

where $L_{i}(X, \sigma)=\frac{\partial}{\partial i} G_{X}(X, \sigma) * I(X)$.

This matrix $M$ has been computed with classical filter, with spherical convolution (10), and spherical Gaussian function (11). Note that in the case of the spherical convolution, since the formula $(f * g)^{\prime}=f^{\prime} * g$ no longer holds, we first have to 
perform the convolution of the spherical Gaussian with $I$, then derive the obtained signal using (3) to obtain $L_{i}$. With our approach, if we consider the relations defined in (17) and (19), $M$ can be easily computed. First, we compare the adapted Harris corner detection with a classical Harris and a Harris computed on the sphere, for the synthetic image proposed in figure (10). We report only the 100 best responses to the detectors. Figure 10(b) corresponds to the detection using classical Harris. We observe some double corner detections whereas some other corners are not detected (17 corners have been detected). Inversely, Harris detection through spherical analysis detects 21 corners but more corners at the image center where there is a blind spot in a real image (Fig 9(a)), but does not detect corners at the periphery (Fig. 10(c)). The proposed method therefore seems being a good compromise (Fig 10(d)), 19 corners have been detected and the results show that the geodesic distance seems capable to correctly detect corners with only slight modifications of the traditional technique. We now verify the behavior of our approach with real images.

Harris detector results are shown for two consecutive real images extracted from a sequence in figure 11 . Table 1 presents a comparison of the repeatability rate developed in [17] between both detectors. If we focus on the particular part in the image which corresponds to the lockers, we can note that 50 corners were detected in $l_{1}$ with the adapted Harris (figure 11(b)) while 55 were found with the conventional approach (figure 11(d)). In the second image, 51 corners were detected with the adapted method while the classical only detected 36 corners. In the classical Harris results, only 29 corners are common between the two images, which represents a repeatability equal to $80 \%$. The geodesic Harris detector presents 43 common corners between images, i.e. a repeatability rate of $86 \%$. 


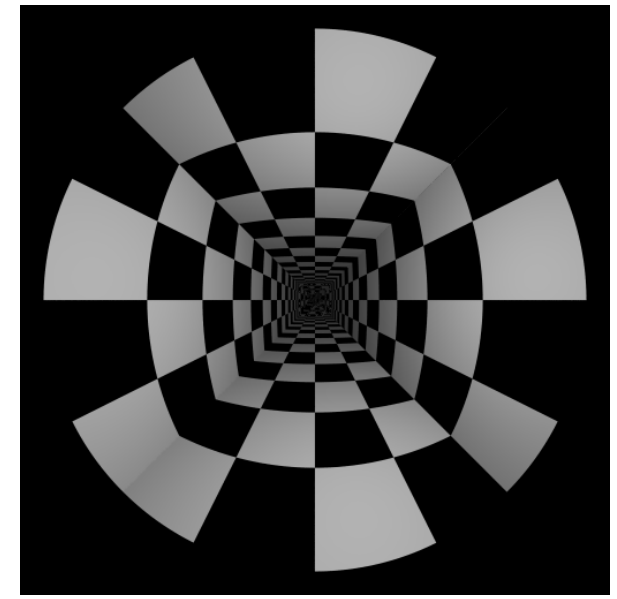

(a)

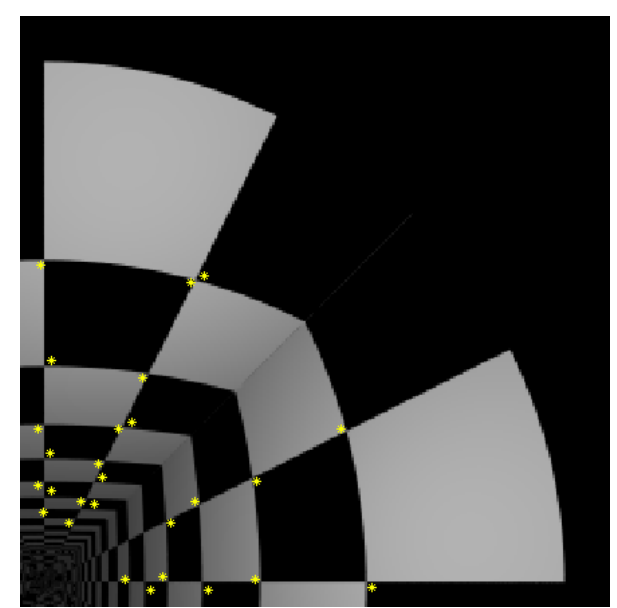

(c)

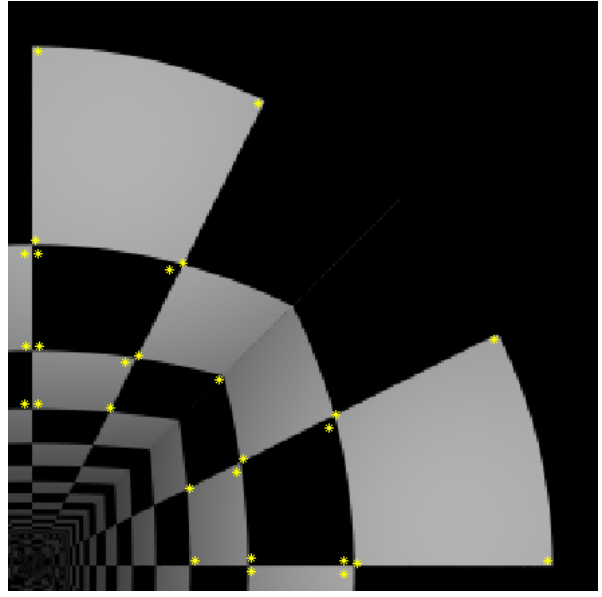

(b)

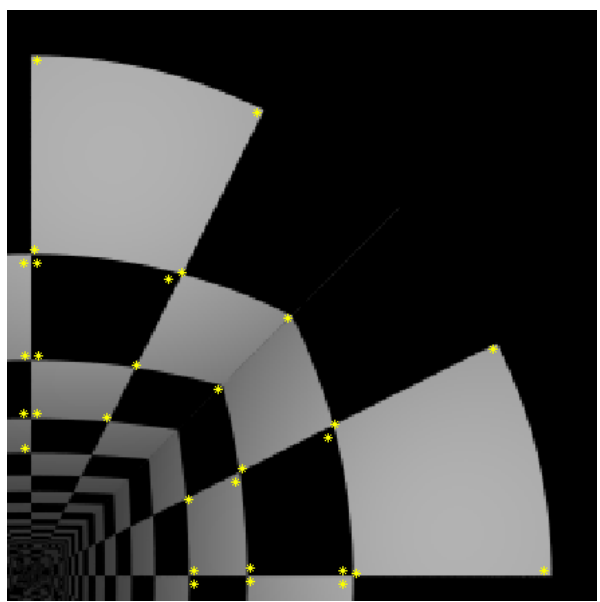

(d)

Fig. 10. Harris corner detection. (a) Test Image, (b) Classical Harris corner, (c) Spherical Harris corner, (d) Adapted method.

\section{Matching}

Feature matching between consecutive images of a sequence is a very important problem in computer vision for motion estimation for example. We are particularly dealing with corner matching, but our approach can be applied to any method based on a local similarity measure. Similarly to image filtering methods, matching techniques must take into account the deformations of the image if they are based on the neighborhood. Let consider the Zero mean Normalized Cross-Correlation function 

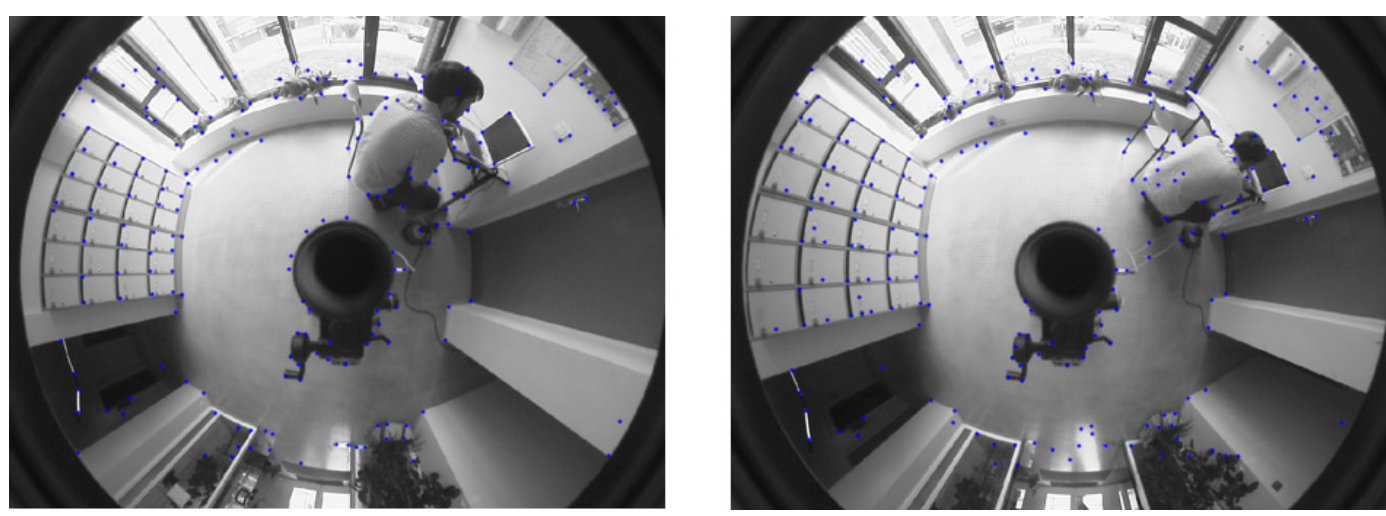

(a)
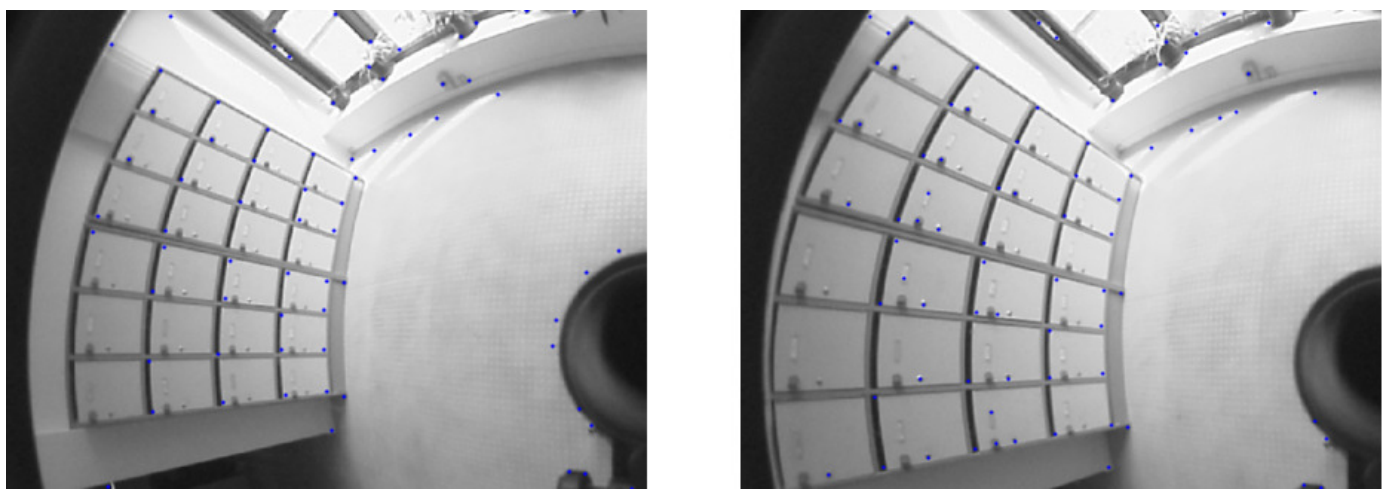

(b)
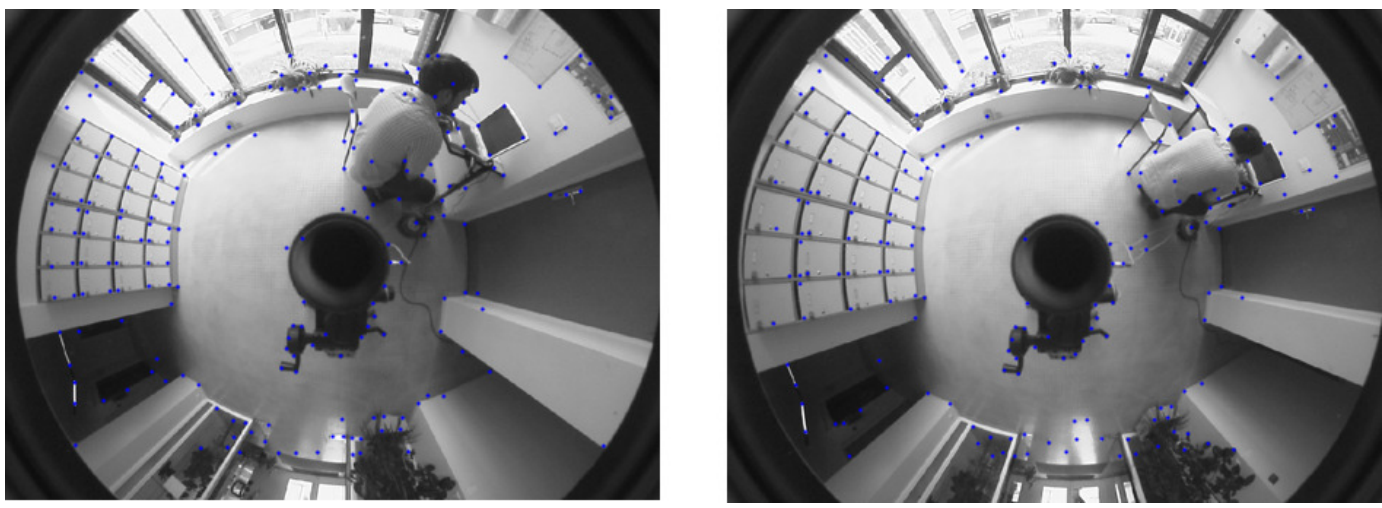

(c)
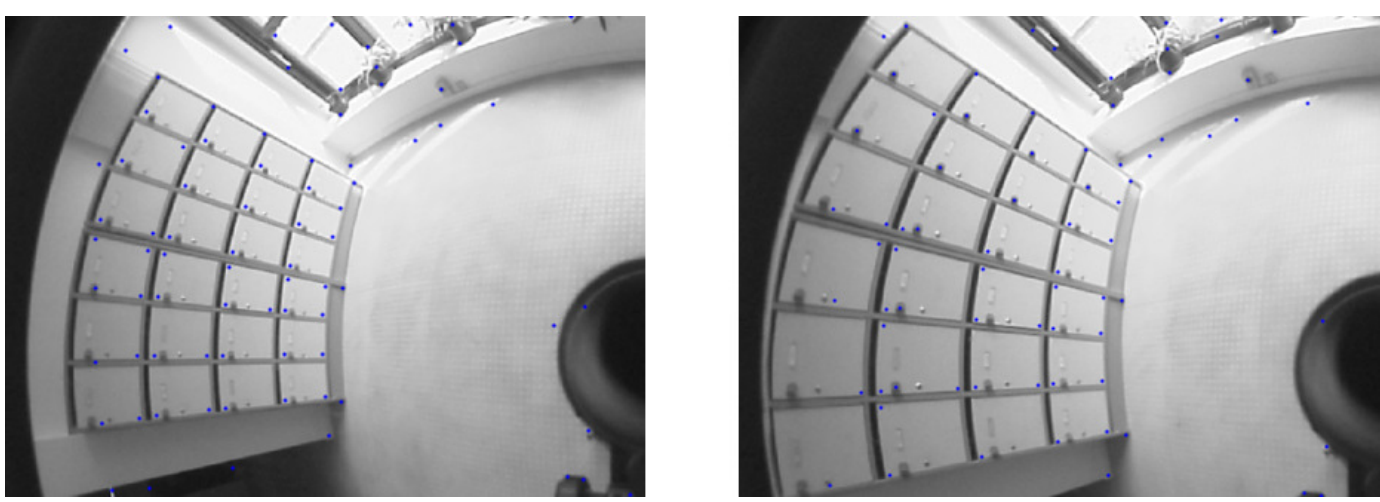

Fig. 11. Harris corner detection results. (a)-(b) Classical method, (c)-(d) Geodesic method. 


\begin{tabular}{|c|r|r|r|r|}
\hline & Adapted Harris method & Classical Harris method \\
\hline Image & $I_{1}$ & $I_{2}$ & $I_{1}$ & $I_{2}$ \\
\hline Corners & 231 & 227 & 212 & 239 \\
\hline Corners on the lockers & 50 & 51 & 55 & 36 \\
\hline Repeatability rate & $43 \rightarrow 86 \%$ & $29 \rightarrow 80 \%$ \\
\hline
\end{tabular}

Table 1

Comparison of Harris method

(ZNCC):

$$
Z N C C(x, y)=\frac{\sum_{i \in \mathcal{V}(x)} \sum_{j \in \mathcal{V}(y)}\left(I_{1}(i)-\overline{I_{1}(x)}\right)\left(I_{2}(j)-\overline{I_{2}(y)}\right)}{\sqrt{\sum_{i \in \mathcal{V}(x)}\left(I_{1}(i)-\overline{I_{1}(x)}\right)^{2} \sum_{j \in \mathcal{V}(y)}\left(I_{2}(j)-\overline{I_{2}(y)}\right)^{2}}},
$$

where $\overline{I_{1}(x)}$ and $\overline{I_{2}(y)}$ are the grey level mean of image $I_{1}$ and $I_{2}$ in neighborhood $\mathcal{V}(x)$ and $\mathcal{V}(y))$, respectively.

For a perspective image, the neighborhood is once again defined from Euclidean distance between points (12). However, for the same reasons previously exposed, this neighborhood can not be applied in catadioptric images. Moreover, a neighborhood on the unitary sphere defined from a regular sampling of the spherical coordinates $\theta$ and $\phi$ is also not adapted. Indeed, such definition does not provide the same neighborhood for each point on the grid (fig 4). Consequently, we also propose to use the geodesic metric to define the neighborhood (16) for measuring the similarity of the detected corners.

We then compare previous images using a classical neighborhood of size $7 \times 7$ and an geodesic neghborhood $\mathcal{V}_{r}^{3}$. If we consider the same corners in both cases obtained by the classical Harris detector with the same thresholds, the use of the 


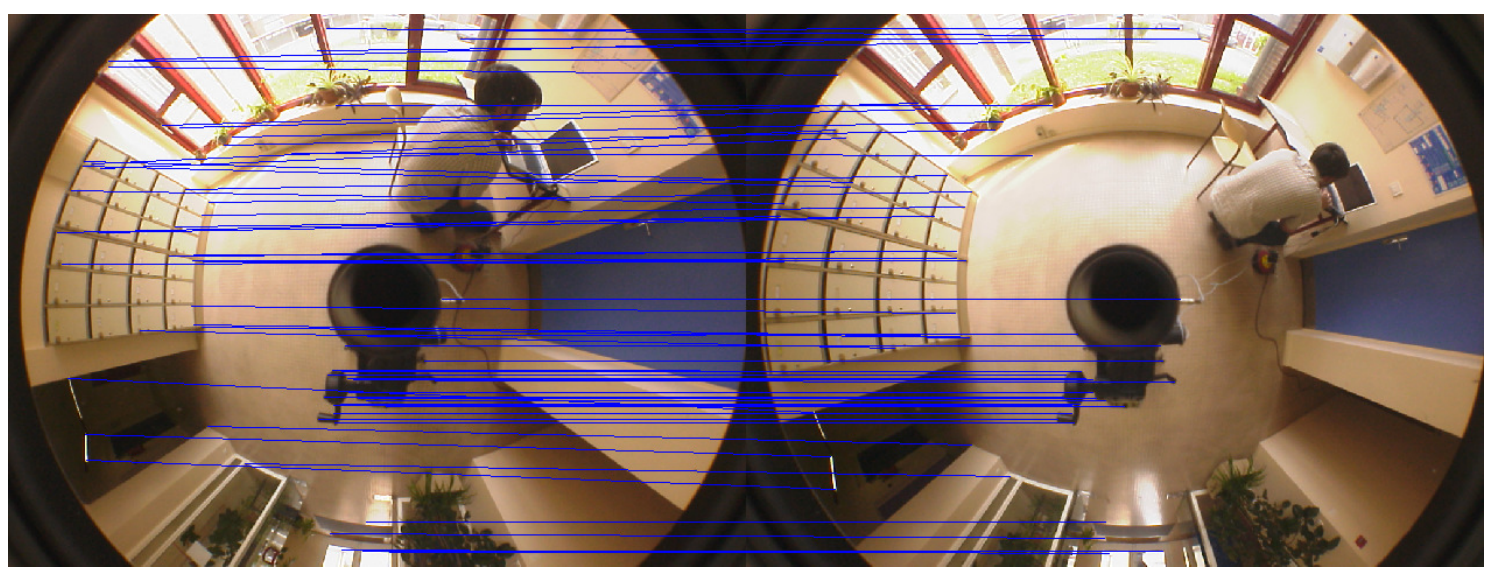

(a)

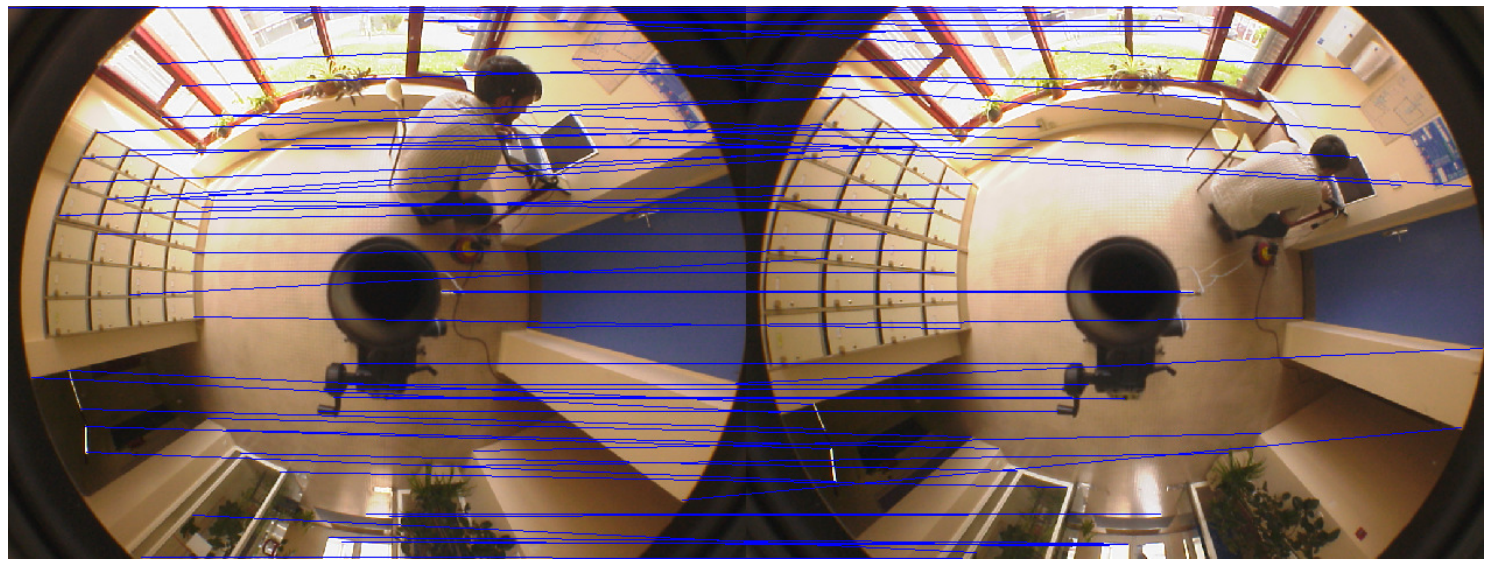

(b)

Fig. 12. Matching by ZNCC. (a) Classical method: 65 total matchings, 53 correct matchings, (b) Geodesic method: 71 total matchings, 63 correct matchings.

classical ZNCC allows to match totally 65 points with 53 correct matchings, which represents a rate of outliers equal to $18.4 \%$. Comparatively, the geodesic neighborhood (16) provides 71 matchings including 63 positive matching, i.e. a rate equal to $11.2 \%$ of outliers (figure 12 ).

Figure 13(c) compares results for corner detection and matching over an outdoor sequence of 21 consecutive images (figures 13(a) and 13(b)). Contrary to the previous matching results obtained from the classical Harris corner detector, we propose here to compare the conventional detection and matching with the adapted detec- 
tion and matching results. In this way, we want to exhibit more significantly the benefits of the geodesic neighborhood. Green bars (respectively red) correspond to the number of matched corners in each image while the blue bars (respectively black) describe the number of outliers for each method. It is worth noting that the geodesic approach provides a better matching since the number of matched corners is greater while the number of outliers is less than for the classical method. Indeed, over the 21 images, the geodesic method provides 152 matchings in average while the classical methods gives 116 matchings. In the same time, the outlier rate is equal to 3.5 in the geodesic case while the classical approach presents a rate of 14.5 . 


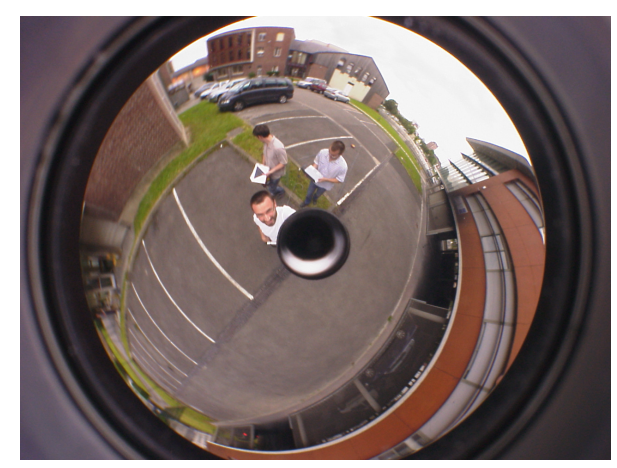

(a)

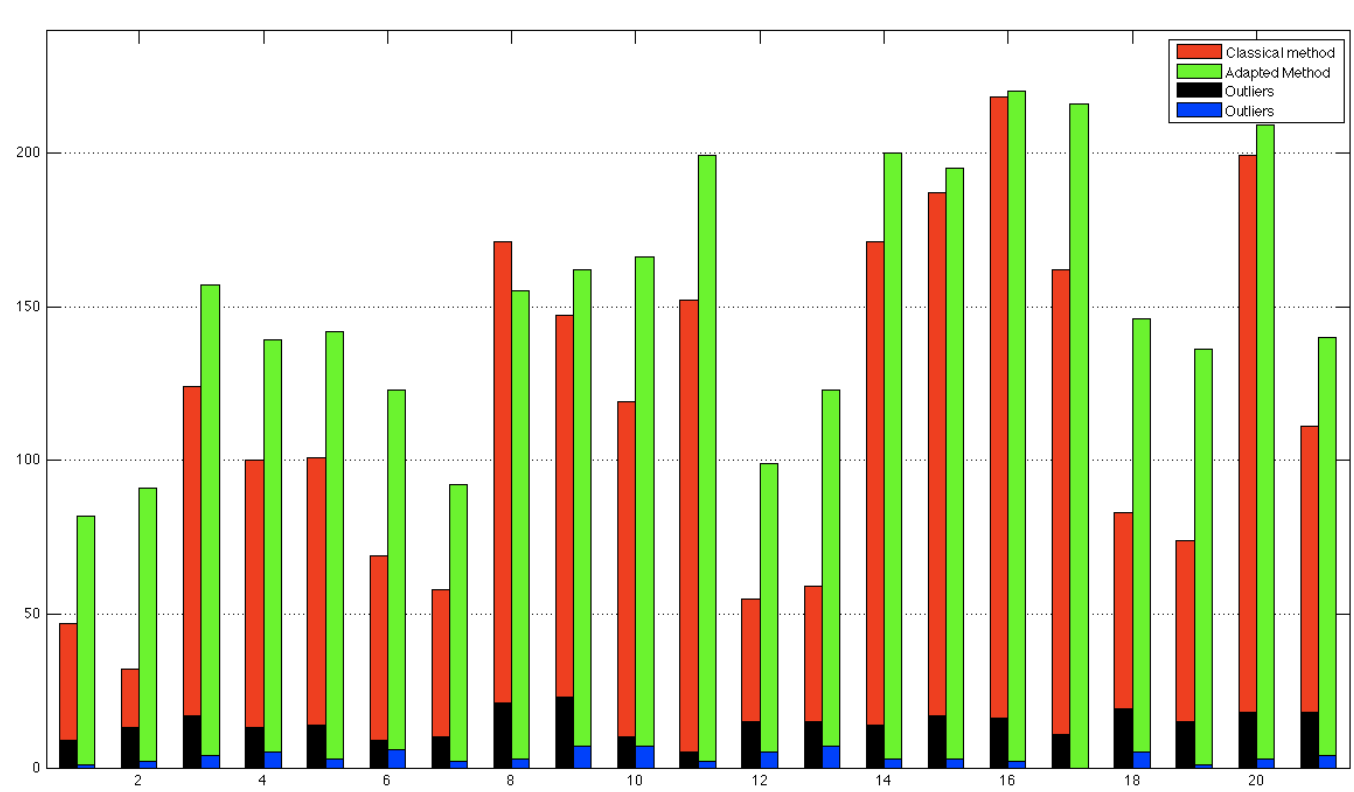

(c)

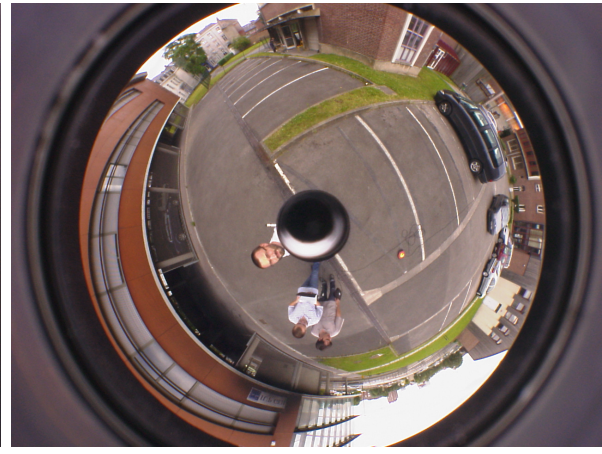

(b)

Fig. 13. Corner detection and matching. (a) (b) 2 images from the real sequence. (c) Matching results.

\section{Conclusion}

In this paper, we deal with central catadioptric image processing. Because of the distortions introduced by the mirror, these images can not be processed as classical perspective images. In this way, we consider a geodesic metric rather the Euclidean one in order to define the dependency neighborhood between pixels. We show that this approach allows to directly adapt the classical perspective image processing 
techniques to central catadioptric images, and more generally to spherical images. Indeed, this geodesic metric allows to apply filtering masks similarly to perspective images while taking into account the distortions of catadioptric images. Experimental results on gradient computation, corner detection, and point matching show a significant improvement of the quality despite the use of the nearest neighbour interpolation. Thanks to this formulation, every method based on pixel mutual dependency can be straightly adapted to central catadioptric images (Markov Random Fields, mathematical morphology, image segmentation...). Moreover, it is worth noting that contrary to methods based on spherical harmonic analysis, we process points only in the image plane. Consequently, pyramidal methods such as proposed by Burt et Adelson [18] remain valid in our approach. It is also important to note that our approach can be applied to any type of single view point sensor, such as perspective camera, central catadioptric camera, but also fish-eye camera [7], since they all provide images equivalent to spherical images. Perspectives will then consist in applying these processing techniques to heterogeneous central sensor network in order to improve their efficiency and interest.

\section{References}

[1] R. Benosman, S. Kang, Panoramic vision: sensors, theory, and applications, SpringerVerlag New York, Inc., 2001.

[2] T. Lemaire, S. Lacroix, Slam with panoramic vision, Journal of Field Robotics 24 (2007) 91-111.

[3] H. Andreasson, T. Duckett, Topological localization for mobile robots using omnidirectional vision and local features, in: In Proceedings of the 5th IFAC Symposium on Intelligent Autonomous Vehicles, 2004. 
[4] I. Bogdanova, X. Bresson, J. Thiran, P. Vandergheynst, Scale-space analysis and active contours for omnidirectional images, IEEE Transactions on Image Processing 16 (7) (2007) 1888-1901.

[5] J. Barreto, A unifying geometric representation for central projection systems, Comput. Vis. Image Underst. 103 (3) (2006) 208-217.

[6] C. Geyer, K. Daniilidis, Catadioptric projective geometry, IJCV 45 (3) (2001) 223243.

[7] X. Ying, Z. Hu, Can we consider central catadioptric cameras and fisheye cameras within a unified imaging model, in: ECCV04, 2004, pp. Vol I: 442-455.

[8] F. Jacquey, F. Comby, O. Strauss, Fuzzy edge detection for omnidirectional images, Fuzzy Sets Syst. 159 (15) (2008) 1991-2010.

[9] C. Demonceaux, P. Vasseur, Markov random fields for catadioptric image processing, Pattern Recogn. Lett. 27 (16) (2006) 1957-1967.

[10] A. Makadia, C. Geyer, K. Daniilidis, Correspondence-free structure from motion, IJCV 75 (3) (2007) 311-327.

[11] S. Bigot, D. Kachi, S. Durand, Spherical edge detector: Application to omnidirectional imaging, in: ACIVS, 2008, pp. 554-565.

[12] K. Daniilidis, A. Makadia, T. Bulow, Image processing in catadioptric planes: Spatiotemporal derivatives and optical flow computation, in: Workshop on Omnidirectional Vision (in conjunction with ECCV 2002), 2002, pp. 3-12.

[13] J. R. Driscoll, D. M. Healy, Jr., Computing fourier transforms and convolutions on the 2-sphere, Adv. Appl. Math. 15 (1994) 202-250.

[14] T. Bülow, Multiscale image processing on the sphere, in: DAGM Symposium Symposium for Pattern Recognition, 2002, pp. 609-617. 
[15] K. Daniilidis, A. Makadia, T. Bülow, Image processing in catadioptric planes: spatiotemporal derivatives and optical flow computation, in: In IEEE Workshop on Omnidirectional Vision, 2002, pp. 3-10.

[16] C. Harris, M. Stephens, A combined corner and edge detection, in: Proceedings of The Fourth Alvey Vision Conference, 1988, pp. 147-151.

[17] C. Schmid, R. Mohr, C. Bauckhage, Evaluation of interest point detectors, International Journal of Computer Vision 37 (2) (2000) 151-172.

[18] P. J. Burt, E. H. Adelson, The laplacian pyramid as a compact image code, IEEE Transactions on Communications COM-31,4 (1983) 532-540. 